\title{
The just-so Higgs story: \\ a response to Adrian Wüthrich
}

\author{
Holger Lyre*
}

July 2010

I give a response to Adrian Wüthrich's critical review of my analysis of the Higgs mechanism, in which I try to clarify some possible misunderstandings. I concede that, as Wüthrich points out, many physicists see the Higgs mechanism as the roll-over from a symmetrical potential in the initial Lagrangian to a symmetry-breaking potential, while my former analysis had basically focused on the gauge-invariant transformation of the initial Lagrangian into the intended form. My main contention, however, still is that neither Higgs story has (as yet) much explanatory power.

\section{Short reprise of the Abelian Higgs Mechanism}

I'm particularly glad for Adrian Wüthrich's critical review (Wüthrich 2010) of my analysis of the Higgs mechanism as presented in my paper "Does the Higgs Mechanism Exist?" (Lyre 2008). For this allows me to clarify some misunderstandings to which my paper - obviously - invites, and for which I am to blame. However, I think I'm not to blame for the core message of the paper. The idea was to shed doubts on an all-too naive picture of the Higgs mechanism as a causal mechanism or "real" dynamical process that takes place in the world rather than a sheer heuristic motivation of the intended GSW electroweak Lagrangian.

Starting point of commonplace presentations of the Abelian Higgs mechanism (the one that is generally used to simplify matters for demonstration) is the Lagrangian

$$
\mathcal{L}^{\prime}=-\frac{1}{4} F_{\mu \nu} F^{\mu \nu}+\left|\left(\partial_{\mu}-i q A_{\mu}\right) \phi\right|^{2}-\mu^{2}|\phi|^{2}-l|\phi|^{4} .
$$

with $l>0$ and $\mu^{2}<0$. This starting point is well-motivated insofar as $\mathcal{L}^{\prime}$ is the most general Lagrangian for a $\mathrm{U}(1)$ gauge invariant renormalizable scalar field $\phi(x)$. Under a polar parametrization of the complex field $\phi=\frac{1}{\sqrt{2}}(v+H) e^{i \frac{\vartheta}{v}}$ with $v=\sqrt{\frac{-\mu^{2}}{\lambda}}$ together with

${ }^{*}$ Philosophy Department, University of Magdeburg, E-mail: lyre@ovgu.de 
the gauge transformation $A_{\mu} \rightarrow A_{\mu}-\frac{1}{q v} \partial^{\mu} \vartheta$ we may rewrite $\mathcal{L}^{\prime}$ as ${ }^{\dagger}$

$$
\begin{aligned}
\mathcal{L}^{\prime \prime \prime}= & -\frac{1}{4} F_{\mu \nu} F^{\mu \nu}+\frac{1}{2} q^{2} v^{2} A_{\mu} A^{\mu}+\frac{1}{2}\left(\partial_{\mu} H\right)^{2}+l v^{2} H^{2} \\
& -\lambda v H^{3}-\frac{1}{4} \lambda H^{4}+\frac{1}{2} q^{2} A_{\mu} A^{\mu} H^{2}+q^{2} v A_{\mu} A^{\mu} H .
\end{aligned}
$$

Since nothing but a re-parametrization of the fields has happened, it should be clear that $\mathcal{L}^{\prime}$ and $\mathcal{L}^{\prime \prime \prime}$ not only obey the same symmetries (Poincaré symmetry as well as global and local U(1) symmetry), but that the physical content of both Lagrangians must be the same. And this, indeed, is no point where Wüthrich and I (or others, for instance Smeenk 2006) disagree. As Wüthrich puts it: "The transition from the system described by the Lagrangian of equation (8) [my $\left.\mathcal{L}^{\prime}\right]$ to the Lagrangian of equation (9) [my $\left.\mathcal{L}^{\prime \prime \prime}\right]$.. is clearly not a transition between two physically distinct systems, as Lyre correctly points out, but a mere transition from one description of the system to another equivalent description."

\section{Wüthrich's worries}

The point where Wüthrich and I disagree is how to identify the Higgs mechanism - and we must see whether this, eventually, is only a trivial point about terminology or perhaps a substantial point. Both of us make clear statements of what we consider to be the Higgs mechanism. But another point first.

On the face of it, the field variables occurring in the Lagrangians $\mathcal{L}^{\prime}$ and $\mathcal{L}^{\prime \prime \prime}$ are different, so one might be tempted to give them differing ontological interpretations. $\mathcal{L}^{\prime}$ describes a system with a complex scalar field $\phi$ and a coupled massless gauge vector field $A_{\mu}$, whereas $\mathcal{L}^{\prime \prime \prime}$ describes a real scalar Higgs boson $H$ with mass $m_{H}=\sqrt{2 \lambda} v$ and a (new) vector field $A_{\mu}$ with mass $m_{A}=q v$. From a mathematical point of view, however, both Lagrangians are on a par, so that neither $\mathcal{L}^{\prime}$ is to be favored over $\mathcal{L}^{\prime \prime \prime}$ nor vice versa. In a note (no. 7) of my 2008 paper I remark that "the reader might perhaps be puzzled by our claims about the equivalence of the three Lagrangians, on the one hand, and the impossibility of a direct realistic interpretation of $\mathcal{L}^{\prime}$ as opposed to $\mathcal{L}^{\prime \prime \prime}$, on the other hand. There seems to be a tension between our first and third observation... And indeed, observation three hinges on stressing a 'quick and literal' interpretation of the particle content of a Lagrangian by simply looking at the mass terms. On the basis of our analysis we may now of course say that such a quick and literal interpretation of $\mathcal{L}^{\prime}$ cannot directly be gained, but is rather indirectly revealed by the direct interpretation of $\mathcal{L}^{\prime \prime \prime} . "$

One thing first about my "third observation" that $\mathcal{L}^{\prime}$ seems to describe a field with a negative mass. As my phrasing already indicates, this only follows from a "quick and literal" (and therefore all-too naive) interpretation of the Lagrangian. I entirely agree with Wüthrich's rigorous explanation from which it becomes clear that such a naive worry is not really justified. But Wüthrich, on the other hand, also agrees that $\mathcal{L}^{\prime}$ compared to $\mathcal{L}^{\prime \prime \prime}$ is "less directly interpretable." So $\mathcal{L}^{\prime \prime \prime}$ is epistemically to be favored.

\footnotetext{
${ }^{\dagger}$ I stick with the notation of my 2008 paper but skip over an unnecessary intermediate step in which $\mathcal{L}^{\prime}$ was transformed into a mathematically equivalent Lagrangian $\mathcal{L}^{\prime \prime}$ and which more explicitly shows how the unphysical Goldstone boson $\vartheta$ becomes absorbed into $A_{\mu}$ due to the above gauge.
} 
Now to our statements about the Higgs mechanism. Whereas I say: "It is precisely this transcription [from $\mathcal{L}^{\prime}$ to $\mathcal{L}^{\prime \prime \prime}$ ] of degrees of freedom-because of the non-invariance of the ground state - which is usually called the "Higgs mechanism", Wüthrich claims: "The Higgs mechanism is the transition from the symmetric to the broken phase in the case of a local symmetry." In his terminology, the symmetric phase is the case of $\mathcal{L}^{\prime}$ with $\mu^{2}>0$, while the broken phase corresponds to $\mathcal{L}^{\prime}$ with $\mu^{2}<0$. So Wüthrich locates the Higgs mechanism in the transition $\mu^{2}>0 \rightarrow \mu^{2}<0$, rather than, as I do, in the transition $\mathcal{L}^{\prime}\left(\mu^{2}<0\right) \rightarrow \mathcal{L}^{\prime \prime \prime}$.

Hence, Wüthrich and I seem to disagree in terminology - so what? Under Wüthrich's terminology "the Higgs mechanism is as real as the phase transition in a ferromagnet." I agree that Wüthrich's Higgs mechanism must be considered a real mechanism. This is a fortiori the case since $\mathcal{L}^{\prime}\left(\mu^{2}>0\right)$ and $\mathcal{L}^{\prime}\left(\mu^{2}<0\right)$ are mathematically and physically different Lagrangians which describe different physical scenarios. And in order to go over from one scenario to the other there's got to be a physical process, a real dynamics which in fact must be represented by a further Lagrangian $\mathcal{L}\left(\mu^{2}>0 \rightarrow \mu^{2}<0\right)$, which as yet has not been made explicit.

The crucial question, however, must be the following: Is it necessary to postulate the transition from $\mathcal{L}^{\prime}\left(\mu^{2}>0\right)$ to $\mathcal{L}^{\prime}\left(\mu^{2}<0\right)$ ? Necessary within the Standard Model? And necessary to explain the existence of the Higgs boson? I don't think so.

\section{My worries}

The problem I have with Wüthrich's understanding of the Higgs mechanism, which might indeed be an understanding shared by many physicists, is that in order to make this "mechanism" not entirely ad hoc or even mystic, one has to tell a detailed story about the causal dynamics driving the transition $\mu^{2}>0 \rightarrow \mu^{2}<0$. Hence, one must present an explicit Lagrangian $\mathcal{L}\left(\mu^{2}>0 \rightarrow \mu^{2}<0\right)$. This is for instance the case in the Landau-Ginzburg model of superconductivity, where one works with a Lagrangian structurally similar to $\mathcal{L}^{\prime}$ but where $\mu^{2}$ has an explicit temperature dependency $\mu^{2}=a\left(T-T_{c}\right)$. And there exists, of course, an additional causal story about temperature change.

In the Standard Model no such story exits. Or at least: the GSW theory in itself doesn't include it. But as long as we do not know where the mystic roll-over $\mu^{2}>0 \rightarrow \mu^{2}<0$ arises from (if it arises at all), the demand of such a transition remains a mere just-so story. Also Wüthrich has only speculations about cosmic coolings to offer. Of course I am fully aware of such speculations. But they are just that: speculations, and therefore carry no weight as explanations of the generation of masses.

Moreover, when I say that Wüthrich's understanding of the Higgs mechanism is perhaps an understanding shared by many physicists, I do not think that all of them share his understanding. Others will share mine. But this shows that the notion of "Higgs mechanism" is indetermined. In their conceptual sloppiness the physicists do not agree in what the reference, the extension of the theoretical term "Higgs mechanism" is. Usually such conceptual sloppiness is absorbed by the precision of the mathematical description. But here things are different. Here it is indetermined which part of the mathematical description belongs to the concept extension: just the derivation from $\mathcal{L}^{\prime}\left(\mu^{2}<0\right)$ to $\mathcal{L}^{\prime \prime \prime}$ or perhaps another, still 
to be spelled out $\mathcal{L}\left(\mu^{2}>0 \rightarrow \mu^{2}<0\right)$ ?

Among those who share my understanding are those, who distribute the most widespread didactic picture and metaphor about the Higgs mechanism. Almost any physicist knows it. It was developed by David Miller to provide a quasi-political explanation of the Higgs mechanism for the former UK Cabinet Minister of Science William Waldegrave. Unfortunately, it is a wrong picture. And, hence, a bad pedagogy for young physicists. Here is the first and main part of it:

Imagine a cocktail party of political party workers who are uniformly distributed across the floor, all talking to their nearest neighbours. The ex-Prime-Minister enters and crosses the room. All of the workers in her neighbourhood are strongly attracted to her and cluster round her. As she moves she attracts the people she comes close to, while the ones she has left return to their even spacing. Because of the knot of people always clustered around her she acquires a greater mass than normal, that is, she has more momentum for the same speed of movement across the room. Once moving she is harder to stop, and once stopped she is harder to get moving again because the clustering process has to be restarted. In three dimensions, and with the complications of relativity, this is the Higgs mechanism. In order to give particles mass, a background field is invented which becomes locally distorted whenever a particle moves through it. The distortion the clustering of the field around the particle - generates the particle's mass.

The workers represent the $\phi$-field of $\mathcal{L}^{\prime}$, a hypothetical background field. The ex-PrimeMinister represents $A_{\mu}$, which couples to $\phi$. Initially, in $\mathcal{L}^{\prime}$, the $A_{\mu}$-field is massless, but when it enters the room (the Universe?) it acquires a mass, as in $\mathcal{L}^{\prime \prime \prime}$. Clearly the picture stresses the transition from $\mathcal{L}^{\prime}$ to $\mathcal{L}^{\prime \prime \prime}$, no attempt is made to mimic the transition from $\mu^{2}>0$ to $\mu^{2}<0$.

Let me come back to Wüthrich's claim that "the Higgs mechanism is as real as the phase transition in a ferromagnet." While I already agreed that under Wüthrich's understanding the Higgs mechanism must indeed be considered a real mechanism, I still want to emphasize (once more) the disanalogy to the ferromagnet. In the case of the ferromagnet the symmetry of the dipoles under the full rotational group $\mathrm{SO}(3)$ is broken to the subgroup $\mathrm{SO}(2)$ by the spin-spin-alignments which prevail around the Curie temperature. Note that in the case of dipole rotations the symmetry possesses real instantiations or, as Healey (2007, p. 152) puts it, the rotational symmetry is an empirical symmetry. A gauge symmetry, however, is no empirical symmetry. In case of the Higgs mechanism the symmetry is only broken on the level of the ground state - by the transition - in Wüthrich's sense - from the bottom of a paraboloid to the brim of a Mexican hat. But why should there be a problem for a ground state not to possess a non-empirical symmetry? (A dangerous question, I know, given the follow-up problem of inequivalent representations in a proper QFT setting.) Ward Struyve (2010) has recently shown what must have been expected to show: that it is possible to give an entirely gauge invariant formulation of the Higgs mechanism. This was Earman's challenge: "Philosophers of science should be asking the ... question: What is the ... gauge invariant structure of the world corresponding to the gauge theory presented in the Higgs mechanism?" (Earman 2004, 1239). By performing a suitable field transformation, Struyve 
manages to separate gauge independent variables from pure gauge ones. This way he gets rid of the unphysical local gauge symmetry and directly arrives at the intended Lagrangian $\mathcal{L}^{\prime \prime \prime}$. Would I have know about this a few years earlier, I probably would not have written my 2008 paper.

On the other hand, the discussion with Wüthrich brings the point about the explanatory (non-) power of the Higgs story to the foreground, which I already pointed out and which should be considered the more substantial point in our discussion than mere controversy about terminology. This point can also be made in the following way: Neither Wüthrich nor I express any reasons to doubt that the Higgs boson, the $H$-field in $\mathcal{L}^{\prime \prime \prime}$, exists. It might very well be that $\mathcal{L}^{\prime \prime \prime}$, or more precisely $\mathcal{L}_{G S W}^{\prime \prime \prime}$, the pendent in the electroweak theory by Glashow, Salam and Weinberg, truly describes reality. And even if one of us would have doubts about the Higgs boson, then those doubts have nothing to do with our disagreement about the Higgs mechanism. But this means that even if the Higgs boson were detected, our disagreement is not settled. However, detecting the Higgs boson means to confirm the last building block of the Standard Model. So the existence of the Higgs boson and the existence of (either Wüthrich's or my) Higgs mechanism are logically and ontologically independent from each other. It might very well be that the former holds in our world while the latter does not (my personal guess is that exactly this will come out in the future, but that's of course not relevant here). It is therefore not necessary to bind the existence of the Higgs boson to a certain, maybe-cosmological process in the world. We simply need no Higgs mechanism, neither in my understanding of the term, nor in Wüthrich's.

Let me end with David Miller's last two sentences in his pictorial explanation to the science minister: "There could be a Higgs mechanism, and a Higgs field throughout our Universe, without there being a Higgs boson. The next generation of colliders will sort this out." I'm afraid, it's exactly the other way round: there could be a Higgs boson, without there being a Higgs mechanism and a Higgs field throughout our Universe, but the next generation of colliders has no chance to sort this out.

\section{References}

Earman, J. (2004). Laws, symmetry, and symmetry breaking: Invariance, conservation principles, and objectivity. Philosophy of Science 71: 1227-1241.

Healey, R. (2007). Gauging What's Real: The Conceptual Foundations of Gauge Theories. Oxford University Press.

Lyre, H. (2008). Does the Higgs Mechanism Exist? International Studies in the Philosophy of Science 22(2): 119-133.

Smeenk, C. (2006). The Elusive Higgs Mechanism. Philosophy of Science 73: 487-499.

Struyve, W. (2010). Spontaneous Symmetry Breaking and the Higgs Mechanism - Lifting the Veil of Gauge Symmetry. Preprint (cf. also philsci-archive.pitt.edu, ID 4728).

Wüthrich, A. (2010). Eating Goldstone bosons in a Phase Transition: A Critical Review of Lyre's Analysis of the Higgs Mechanism. Preprint, philsci-archive.pitt.edu, ID 5496. 